\title{
Investigating Collaborative Annotation on Slate PCs
}

\author{
${ }^{1}$ Jennifer Pearson, ${ }^{2}$ George Buchanan, ${ }^{1}$ Harold Thimbleby \\ ${ }^{1}$ Swansea University, Swansea \\ csjen@swan.ac.uk, csharold@swan.ac.uk \\ ${ }^{2}$ City University, London \\ george.buchanan.1@ city.ac.uk
}

\begin{abstract}
Mobile reading is becoming evermore popular with the introduction of eInk devices such as the Kindle, as well as the many reading applications available on slate PCs and cellular handsets. The portable nature and large storage capacity of these modern mobile devices is making reading a more technology orientated activity. One aspect of mobile reading that has been given surprisingly little attention is collective reading - which is a common activity with paper documents. We investigate the support of group reading using slate PCs, focussing on collective annotation. In the past, desktop PCs have proved inferior in many ways for reading, when compared to paper. Notably, user evaluations of our new system, BuddyBooks, demonstrate that the slate PC form factor can, in contrast, provide advantages for group reading. While annotation practices change with the new format, coordinating within the group can be improved when touch-interaction is carefully exploited.
\end{abstract}

\section{Author Keywords}

Documents, Annotation, Collaboration, Slate PCs

\section{ACM Classification Keywords}

H.5.2 [User Interfaces]: input devices and strategies; interaction styles; prototyping

\section{INTRODUCTION AND BACKGROUND}

While twenty years ago it was the norm to read on paper, now it is a common sight to see people reading from portable devices such as eReaders, slate PCs, and even cellphones. There has been a large body of work dedicated to reading from both mobile and desktop devices. Reading on small screens was investigated as far back as the early 80s [3], and the popularization of online web browsing and reading led to an initial surge in mobile reading over a decade ago [2]. In that context, the main focus was on web content, much of which is relatively brief. Research has demonstrated that both back-lit displays (of all sizes), and small displays are associated with shorter spans of reading. In general close, attentive reading is rare on digital media, even on larger displays [5].

Permission to make digital or hard copies of all or part of this work for personal or classroom use is granted without fee provided that copies are not made or distributed for profit or commercial advantage and that copies bear this notice and the full citation on the first page. To copy otherwise, or republish, to post on servers or to redistribute to lists, requires prior specific permission and/or a fee.

MobileHCI '12, September 21-24, 2012, San Francisco, CA, USA.

Copyright 2012 ACM 978-1-4503-1105-2/12/09...\$10.00.
There has also been an interest in devices that support prolonged attentive reading. Researchers in "appliance design" investigated the usability of reading appliances [6] - the forerunners of contemporary reading devices, such as the Kindle and iPad. A recent resurgence in reading devices has resulted in research on their legibility and general usability (e.g. [9]). However, this has tended to focus on display technology, rather than interaction design. Another question has emerged around input technology; prototype reading appliances used a stylus, whereas modern devices often adopt touch. The different affordances of the two technologies have provoked a question about their relative merits for different tasks. Annotation is a central task to attentive reading, and the suitability of touch-sensitive devices for annotation [4] has emerged as a key research question.

Research has often focussed on the solitary reader [5]. That emphasis is repeated in the initial designs of reading devices. While reading can be alone, reading with others is surprisingly pervasive: e.g., when school children are encouraged to read in groups. In a mobile context, web content can be shared and read among friends by passing around a smartphone. There are also more structured examples of group reading, such as acting rehearsals, book clubs and research groups.

It seems to be an appropriate time to investigate collaborative reading on mobile devices, particularly those devices that are frequently used for longer spans of reading. While social annotations have started to emerge in the Apple iBooks and Kindle Reader software, reading that is shared in the same time and place is not directly supported, despite it being a commonplace activity. There is limited support for multiple screens, shared mark-ups or group coordination within a document. Some commercial whiteboard applications let users work in real-time, on multiple devices making changes to a common document, but these (often being aimed at classroom lecturing) are not reading focused and do not address the problem of coordination.

Electronic media have proved ineffective for certain styles of reading. In collaborative group reading, multiple paper documents remain a popular choice as paper is easily manipulated, and the physical form allows users to sit wherever space is available and interact with each other without being constrained by bulky technology. Individual copies faciliitate personal notes - which would prove difficult if several people shared one single copy.

In this paper, we focus on co-located collaborative reading, as opposed to remote reading, in consequence of initial research [8] that investigated the social context of that activity in detail, 
drawing a contrast between digital and printed media. The study of users' current behaviour allowed us to narrow and focus the scope of the work undertaken here. However, we also drew on a range of prior research from mobile HCI.

Social and public annotation is a popular topic of research in the mobile context [1], but typically the focus lies outside the specific activity of reading (e.g., photos). In contrast, investigations into the annotation of text have focussed on the use of both desktop PCs and paper, and overlooked mobile devices [5]. Annotations created during reading often contain a high proportion of text. It is well known that typing is a problem on mobile devices, though much improved in recent years. The impact of reduced screen size and the heightened cost of typing on annotation behaviour has received minimal attention, though differences would certainly be expected.

\section{BUDDYBOOKS: A COLLABORATIVE READING SYSTEM}

This paper describes BuddyBooks, a mobile-based real-time application for collaborative reading in groups. Each member of the group uses their the BuddyBooks app on a personal iPad, mimicking the way groups work together using multiple paper documents. The slate PC form factor allows users to sit near each other, while giving each a personal document view, and providing an intimate social interaction in the shared task. Our software allows each group member to mark-up their own copy of the document while simultaneously viewing the notes of other members. The new interface includes a tool that allows users to quickly and easily 'point out' specific parts of a document to others, helping the group coordinate its reading. Figure 1 shows examples of the tools available within the BuddyBooks application.

\section{System Architecture}

To use BuddyBooks, each client iPad must first connect to the host server sending it their IP address and nickname. The server allocates each user a unique color which is used to distinguish between the activity of the readers. The server receives notifications of any mark-ups made, and forwards that information to all the other devices. The document itself is stored locally on the iPads, but the server also keeps a record of the document as well as all the changes made to it for late joining group members to receive all the information. The purpose of BuddyBooks is to allow users to collaboratively read a common document. It is not therefore, concerned with the collective editing of a document but rather as an aid to mark-up and co-ordination on content where the original text remains constant.

\section{Annotation Tools}

Our goal for BuddyBooks was to allow users the same freedom to mark-up that paper offers while simultaneously overcoming paper's limitations. With this in mind, we opted to include a set of document annotation tools for use within the BuddyBooks interface. The three main objectives we had in mind for these tools were: they should have the same key functions of paper (i.e., users must be able to highlight, bookmark and make notes); they notes of all group members must be visible in real-time by every other group member; and different member's notes must be distinguishable. As a first

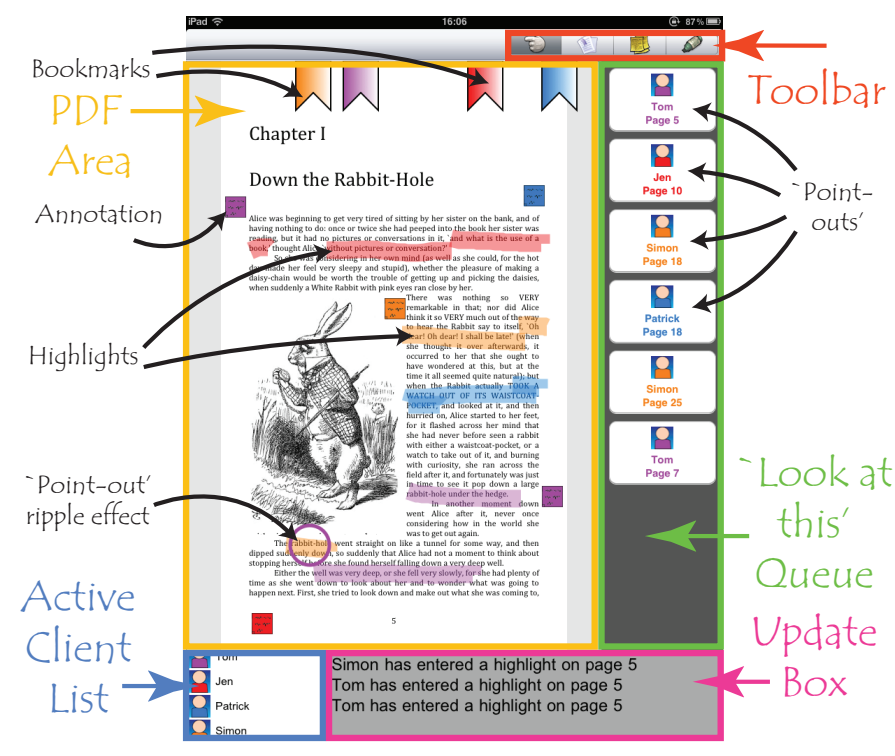

Figure 1. Screen shot from BuddyBooks

cut implementation then, we included three main annotation tools in our interface (see Figure 1) : a bookmark tool (shown as ribbons on the top of a page - four are visible in this example); a note-taking annotation tool (shown as small squares on the document view); and a highlighter tool (shown as semitransparent colored lines). These are all activated by touch gestures. Clicking anywhere on the page when in bookmark mode adds or removes a bookmark for the current page. When an annotation box is clicked, an on-screen keyboard is made visible to add, edit or delete annotations. When highlighting, dragging across the document page leaves a colored trail. All marks made on the working document are sent immediately to every iPad in the group. Each member's marks is distinguished by a unique color.

\section{'Look at This' Queue}

Often in a group collaboration session, users will want to indicate items and locations to other group members, (e.g., "look here on page 10, paragraph 2"); something which we call mutual navigation. If all members of the working group have their own copy of the document, it can sometimes be tricky and time consuming for everyone to locate the exact spot in the document, which can consequently cause a break in attention.

To support this, BuddyBooks has a 'look-at-this' queue that allows users to 'point out' specific areas of the original document to other members of the group. Essentially then, the look-atthis queue is a list of temporary placeholders that allows group members to quickly and easily navigate to a specific area of the working document.

To point out a location in the document, a user must simply select the tool and touch on the spot they wish the others to look at. This then automatically sends a point-out to the top of all group members look-at-this queue along with the color and nickname of its creator. Other group members are 
now aware they are being summoned to another part of the document but can wait to click on the hyperlinked point-out until it is convenient for them. Clicking on an entry in the queue navigates the user to the correct document page and animates a ripple effect (a circle outline that pulses in and out around the co-ordinate point for several seconds) to indicate to the user the exact page and location the point-out was made.

\section{EVALUATION}

To evaluate how well BuddyBooks performs compared to traditional paper-based group reading, we performed a comparison study using 18 participants (10M, 8F) aged between 22 and 61. These participants were selected from a cohort of staff and students from Swansea University who have previously undertaken collaborative reading in groups. We split these participants into groups of 3 and 4 providing us with 5 reading groups who then performed reading tasks interactively together. The purpose of the study was to observe participant behaviour while completing each of the group reading tasks and to obtain subjective feedback on each of the methods. These reading sessions took on average, around an hour and a half to complete and each participant was given a $£ 10$ gift voucher in return for their time.

Each group session began with the participants completing a questionnaire on their current group reading practices. This was followed by a comprehension-style reading task on a set of paper-based documents (one per participant). Readers were encouraged to discuss and mark-up their documents and were given a range of stationary with which to do so. The goal of the group reading task was to identify weaknesses and errors in the text, to mark these on the document along with a short summary at the end. Immediately following this task, the users then performed a similar task on a different document using BuddyBooks.

We used two documents, of a similar length and complexity, but differing in topic to avoid learning effects. To ensure an unbiased experimental design, we alternated the order these documents were presented to each group. Finally, after the study, each participant was asked to fill out a second questionnaire to gather subjective information regarding each of the respective systems.

The pre-study questionnaire revealed that 11 of the 18 users regularly participated in collaborative reading sessions (the remainder do it occasionally), and the primary medium for 14 participants was paper. The other 4 reported using a computer as their primary medium; none made use of mobile devices such as iPads or Kindles. Given the current popularity of portable reading devices, we feel this statistic highlights the lack of support available for using them for collaborative reading.

The documents used by the participants were two current affairs issues of social concern, with these being used in a balanced pattern between groups for the two different media i.e. paper and BuddyBooks. None required specific domain knowledge, but each included detailed and complex argument, and was intended for an audience with strong reasoning skills. The groups were asked to critique each paper, and mark key points as they individually saw fit.

\begin{tabular}{|l||l|l|l|l|l|}
\hline \multicolumn{1}{|l||}{} & \multicolumn{2}{|c|}{ Paper } & \multicolumn{2}{c|}{ BuddyBooks } & Mann- \\
& Avg & SD & Avg & SD & Whitney \\
\hline $\begin{array}{l}\text { Q1: How easy was it to } \\
\text { view notes made by other } \\
\text { members of the group? }\end{array}$ & 4.5 & 2.4 & 8.6 & 1.5 & $\begin{array}{l}\mathrm{p}<0.0005 \\
\mathrm{U}=298.5\end{array}$ \\
$\begin{array}{l}\text { Q2: How easy was it to cre- } \\
\text { ate notes? }\end{array}$ & 8.2 & 2.3 & 7.9 & 1.7 & $\mathrm{X}$ \\
$\begin{array}{l}\text { Q3: How easy was it to } \\
\text { highlight a section of a doc- } \\
\text { ument and share it with all } \\
\text { members in the group? }\end{array}$ & 5.3 & 2.4 & 9.1 & 1.1 & $\begin{array}{l}\mathrm{p}<0.0005 \\
\mathrm{U}=297.5\end{array}$ \\
$\begin{array}{l}\text { Q4: How easily could you } \\
\text { point out bits of information } \\
\text { to one another? }\end{array}$ & 5.06 & 2.29 & 8.17 & 1.62 & $\begin{array}{l}\mathrm{p}<0.0005 \\
\mathrm{U}=281.5\end{array}$ \\
$\begin{array}{l}\text { Q5: What score would you } \\
\text { give each system overall? }\end{array}$ & 5.67 & 2.25 & 8.56 & 1.34 & $\begin{array}{l}\mathrm{p}<0.0005 \\
\mathrm{U}=283\end{array}$ \\
\hline
\end{tabular}

Table 1. Average subjective ratings (out of 10). $\mathrm{X}$ indicates a nonsignificant result.

\section{Results}

We asked the participants to rate each system (paper and BuddyBooks) out of 10 (10 being highest) for a range of attributes relating to group reading. Table 1 shows a selection of these ratings.

The participants' subjective responses show a strong preference for BuddyBooks compared with paper with average overall ratings (Q5) producing significant (Mann-Whitney) results of 5.67 and 8.56. These promising results were also supported by participants' comments:

"Real time, multiple people editing is major plus point. I
would love to adopt this over the reams of paper"

"It's good to see new system/technology and to see something working well, and how it could one day help me in my job"

We asked users to rate how easy it was to view notes made by other members of the group (Q1), how easy it was to create notes of their own (Q2) and how easy it was to create highlights and share them with all other group members (Q3). Q1 and Q3 here, both yielded highly significant subjective scores in favour of BuddyBooks, suggesting it provides a marked improvement over paper for collaborative reading. Some of the reasons as stated by the participants as why they preferred BuddyBooks included: more legible notes, real-time updates, variety of easy functions and it doesn't require pens. Of these reasons, the ability to see other's notes on their own document seemed to be the most popular feature prompting comments such as:

"It's very easy to see what others are doing with their annotations/highlights."

"It was good that I could see other's highlights of spelling mistakes then there was no need for me to mark it too. Saves us all doing the same job."

"Being able to see everyone's notes in their own color helped a lot I knew that blue comments we made by someone else than the red ones, I could directly ask people in case of any questions." 
Though participants did not find it significantly easier to create notes using BuddyBooks, the results for this were relatively high for both systems, indicating that the new interface did not add any novel problems.

With regards to the form factor of slate-PCs, participants' comments were generally positive in terms of their wireless form, size, shape which allows them to be used much in the same way as a clipboard and pen.

Turning our attention to mutual navigation, which, as already noted, can be problematic during group reading on paper, Q4 shows that users found it easier to point out content to each other using BuddyBooks rather than paper. The observed behaviour of the participants also uncovered a surprising difference between the two interfaces. Specifically, there was a lot less time spent discussing where in the document to look in BuddyBooks than they did on paper (i.e., in the paper task, all five groups were heard describing where to look - e.g., "over here on page 10, half way down paragraph 2"). Thus, a much larger number of comments were being made about the content of the text itself in BuddyBooks, and when using paper a large amount of discussion concerned where in the document to focus. BuddyBooks appears to encourage discussion within collaborative working groups - a valuable contribution that demonstrates the value of slate PCs for digital collaborative reading.

One issue that particularly concerns us is the relative use of different styles of annotation when using this form-factor. Highlighting was used extensively (just under $75 \%$ of all marks), and annotations and bookmarks only minimally (5\% and 3\% respectively). The novel point-out tool accounted for the remaining 17\%. For example, group 1 used 13 point-outs, 66 highlights, 5 annotations and 2 bookmarks. The use of these different methods on paper and desktop PCs is well documented. Typical values on paper would usually have at least double (10\% to $35 \%)$ the number of textual notes [5] - and thus it appears participants reduced their use of written notes.

The volume of markups between our paper and iPad modes was similar, and both corresponded with documented paper behaviours. Nonetheless, there remains some caution about the iPad form factor for a number of participants, prompting comments such as:

"you can write corrections more easily [on paper]."

"sometimes its nice to do things by hand."

In digital reading the volume of annotations of all types is typically low - a sixth to a third of the number found on paper. In contrast, the volume of markup was consistent between paper and BuddyBooks. We thus have overall annotation behaviours that mirror the volume of paper, and exceeds that of desktop work, but which emphasises non-textual marks over written notes.

\section{CONCLUSIONS}

Seymour Papert [7] commented that a primary school teacher 'teleported' from a thousand years ago would notice hardly any difference. Had he used the term "collaborative reading" this would be one of the things that has not changed noticeably. As this paper emphasizes, today's technology can change how collaborative reading is performed - it can enhance mobility and coordination.

The BuddyBooks prototype has shown the utility of slate PC devices for collaborative group reading. When using a midsized touch-screen device, the participants in our experiment demonstrated a high level of annotation - a contrast to the normal characteristics of electronic reading on the desktop. Using a PC results in few annotations and a low level of interaction with the text. However, there were changes and adjustments to the participants' use of annotation facilities textual annotations were infrequent, while highlighting was commonplace.

We have also demonstrated the specific advantages of mobile devices to enhance reading together. Our support for coordinating reading, which avoids the verbose description of document content and locations, demonstrated the potential of mobile technology to expand the scope of reading beyond traditional media. This work is a companion paper to another body of research into collaborative reading [8].

\section{ACKNOWLEDGEMENTS}

This work was supported by Microsoft Research Cambridge, and EPSRC grants EP/F041217 and EP/G003971/1.

\section{REFERENCES}

1. M. Ames and M. Naaman. Why we tag: motivations for annotation in mobile and online media. In Procs CHI 'O7, pages 971-980. ACM, 2007.

2. G. Buchanan, S. Farrant, M. Jones, H. Thimbleby, G. Marsden, and M. Pazzani. Improving mobile internet usability. In Procs WWW'01, pages 673-680. ACM, 2001.

3. R. L. Duchnicky and P. A. Kolers. Readability of text scrolled on visual display terminals as a function of window size. Human Factors, 25:683-692, 1983.

4. G. Golovchinsky, S. Carter, and A. Dunnigan. Ara: the active reading application. In Procs $M M^{\prime} 11$, pages 799-800. ACM, 2011.

5. C. C. Marshall. Reading and Writing the Electronic Book. Synthesis Lectures on Information Concepts, Retrieval, and Services. Morgan \& Claypool Publishers, 2009.

6. C. C. Marshall, M. N. Price, G. Golovchinsky, and B. N. Schilit. Introducing a digital library reading appliance into a reading group. In Proc. DL '99, pages 77-84. ACM, 1999.

7. S. Papert. The children's machine: rethinking school in the age of the computer. Basic Books, Inc., New York, NY, USA, 1993.

8. J. Pearson, T. Owen, H. Thimbleby, and G. Buchanan. Co-reading: Investigating collaborative group reading. In Procs of JCDL '12, page In Print. ACM, 2012.

9. E. Siegenthaler, P. Wurtz, and R. Groner. Improving the usability of e-book readers. J. Usability Studies, 6:3:25-3:38, November 2010. 